\title{
Creating an Online World Flora by 2020: a perspective from South Africa
}

\author{
Janine E. Victor • Gideon F. Smith • Nicholas J. Turland • \\ Marianne le Roux • Alan Paton • Estrela Figueiredo $\bullet$ Neil R. Crouch • Abraham E. \\ van Wyk • Denis Filer • Erich van Wyk \\ J. E. Victor (correspondence author), G. F. Smith, M. le Roux , E. van Wyk \\ Biosystematics Research and Biodiversity Collections Division, South African National Biodiversity \\ Institute, Private Bag X101, Pretoria 0001, South Africa \\ e-mail: j.victor@sanbi.org.za \\ G. F. Smith, E. Figueiredo \\ Departamento de Ciências da Vida, Centre for Functional Ecology, Universidade de Coimbra, 3001 - \\ 455 Coimbra, Portugal

\section{N. J. Turland} \\ Botanischer Garten und Botanisches Museum Berlin-Dahlem, Freie Universitat Berlin, Konigin-Luise- \\ Str. 6-8, 14195 Berlin, Germany
}

A. Paton

The Herbarium, Royal Botanic Gardens, Kew, Richmond, Surrey TW9 3AB, UK

E. Figueiredo

Department of Botany, Nelson Mandela Metropolitan University, PO Box 77000, Port Elizabeth 6031, South Africa

\section{N. R. Crouch}

Ethnobotany Unit, South African National Biodiversity Institute, Berea Road, P.O. Box 52099, Durban 4007, South Africa

\section{N. R. Crouch}

School of Chemistry and Physics, University of KwaZulu-Natal, 4041 Durban, South Africa

D. Filer

Department of Plant Sciences, University of Oxford, South Parks Road, Oxford OX1 3RB, UK

\begin{abstract}
At the 10th Conference of the Parties of the Convention on Biodiversity (CBD), which was held in Nagoya, Japan, in October 2010, an updated Global Strategy for Plant Conservation (GSPC) was adopted as part of the plan of work of the CBD. Target 1 of the GSPC aims to produce an online Flora for all the plants of the world by 2020 . Governments that have ratified the CBD will have to report over the next several years on progress towards achieving this challenging target. Floras are still widely regarded as a means of providing descriptive information and identification tools for the plants that occur in a specified region. Historically, Floras have included identification keys; scientific names with authorship for all taxa known to occur in the area; synonymy; descriptions; distributions within the region in question; specimen citations; habitat; literature references; and illustrations. Of these, nomenclature, descriptions, identification tools, illustrations and distributions are critical components. The approach being taken by South Africa, a biodiversity-rich country, in working towards achieving Target 1 of the GSPC by 2020 is presented and discussed, outlining a methodology that may be of practical use to other countries. We
\end{abstract}


hope this will urge other countries to consider how they might meet this challenging conservation target.

\section{Keywords}

BRAHMS, Convention on Biodiversity, Flora, Global Strategy for Plant

Conservation, Online, South Africa, Taxonomy, Vascular plants

\section{Introduction}

In 1999, the international botanical community attending the XVI International Botanical Congress in Saint Louis, MO, United States, resolved that plant conservation should be regarded as a priority in biodiversity conservation. This led to the development of the Global Strategy for Plant Conservation (GSPC) within the framework of the Convention on Biodiversity (CBD) in 2002 (COP 2002). The first target of the GSPC as then construed was for the plant taxonomists of the world to develop a working list of all known plant species by 2010. Through a collaborative effort between the Royal Botanic Gardens, Kew, the Missouri Botanical Garden and many contributors, a global plant checklist was placed online in December 2010 (The Plant List 2010). A second and updated version of this list is scheduled to be released soon, correcting errors detected in the first version, including further datasets, and reducing the number of unresolved names. Four years earlier than the target deadline, South Africa had published a comprehensive country-level checklist (Germishuizen et al. 2006), enhanced with primary biological information such as plant height, growth form, and altitudinal range occurrence. This volume was a successor to earlier regional floristic checklists that first appeared from the 1980s to the 1990s. These publications reflected the cumulative work of several generations of collectors, taxonomists, recorders and databasers and were built on extensive regional herbarium collections. An update of the GSPC for the decade spanning 2011-2020 was adopted by the 10th Conference of the Parties of the CBD in Nagoya, Japan, in October 2010 (COP 2010). Sixteen outcome-orientated targets were specified to achieve five objectives by 2020. Target 1 of the updated GSPC is to build on the success of the 2010 Target 1 by creating " an online flora of all known plants' by 2020 (COP 2010). A resolution of the XVIII International Botanical Congress in Melbourne, Australia, in July 2011, called for "botanical institutions worldwide to collaborate to achieve a comprehensive and authoritative 'online flora of all known plants' by 2020"' (IBC 2011). In this way, the international conservation and botanical communities have acknowledged that taxonomy provides essential baseline information that is a prerequisite to the success of all other targets. A widely accessible online Flora (with a capital ' $F$ ' denoting a hardcopy or electronic publication on the plants of a region) of all known plant species is critical in plant and ecosystem conservation efforts. South Africa, which is recognised as one of the megadiverse countries of the world, has a flora of about 20,000 indigenous and naturalised plant taxa (Steenkamp and Smith 2006). In this paper, we explore possible ways in which South Africa, and conceivably countries in the rest of the southern African subcontinent and elsewhere, can achieve Target 1 of the GSPC 2020, after having successfully responded to Target 1 of GSPC 2010. 


\section{End-users of Flora information}

In the year before October 2013, the website of The Plant List (2010) was visited more than 2 million times by a large audience of 1.1 million users from all walks of life. Even though the list does not reflect a consensual, authoritative classification, it is used to provide immediate information on the status of plant names and as a tool to access further information on those names in 14 linked websites. This level of use is an indication of the potential success of a more comprehensive product such as an online Flora.

Given the scope of the 16 GSPC 2020 targets, the potential users of a World Flora Online (WFO) will have a broad range of interests and requirements for information (Paton 2009). The WFO therefore needs to provide the necessary taxonomic information, but also link to a broad range of other facts about plants. Names and synonymy, distribution data, identification aids and cross-referencing to sources such as the Encyclopaedia of Life (EOL) and the Global Biodiversity Information Facility (GBIF) are likely to be priorities for the implementation of biodiversity policies (Smith 2005). Policy implementation will require institutional support and collaborative taxonomic initiatives to deliver required information in appropriate formats (Kirkup et al. 2005; Smith et al. 2008).

Target 1 of the GSPC clearly underpins the other Targets. The most obvious example of this is that Target 2, "An assessment of the conservation status of all known plant species", cannot be achieved without a reliable list of species to build on.

Achievement of other species-orientated targets such as Targets 7, 8, 9, 10, 11 and 12 will depend on knowing which species exist and where, while habitat-orientated targets such as Targets 4, 5 and 6 will require knowledge of the species within specific geographic areas to assist in conservation planning and monitoring. As the WFO is a collaborative endeavour, it will also support Targets 15 and 16 that deal with training and strengthened collaborations.

The WFO is not only important to achieve the GSPC targets, but also for implementing the CBD in general (CBD 2013), including broader biodiversity goals such as the Aichi Targets and the Millennium Development Goals (MDG). Within the $\mathrm{CBD}$, the need for taxonomy and the taxonomic expertise necessary to implement the convention has been recognised as the Global Taxonomy Initiative (Klopper et al. 2002; Smith et al. 2008). The WFO, providing a taxonomic baseline for the plant components of biodiversity, needs to support the synthesis of information necessary to implement relevant CBD decisions and support broader biodiversity goals with specific emphasis on conservation.

\section{Challenges in achieving a World Flora Online}

Before building a WFO it is necessary to have or to compile a list of names to which floristic information can be linked. How to resolve the problem of nomenclatural consensus between countries at an international level is probably the most challenging obstacle faced by compilers of the WFO. For example, the South African National Biodiversity Institute (SANBI) has taken a decision to follow the Angiosperm Phylogeny Group (APG) II classification system, whereas a European and Australian consortium, the Vascular Plant Classification Committee, has taken a decision to 
follow the more recent APG III system (Wearn et al. 2013). At or below genus level this problem is not insurmountable, if all floristic information for the WFO is databased using the genus name as the searchable point of reference rather the than family name. Ideally the database should enable more than one family name to be linked to the genus name so that different countries can use alternative classification systems. For country endemics, data can be relatively easily incorporated into a global flora. For non-endemic species the task is much more complex, for example, there may be inconsistency in which genera or species are accepted in different areas. Such instances of incongruence between different taxonomic views can help prioritise further taxonomic work. National contributions, expertise and classificatory bestpractise should inform global consensus taxonomy. The global overview provides information that is not available nationally, such as global context for species delimitations and distributions. This interplay between national and global levels is critical to the success of the GSPC (Paton and Nic Lughadha 2011).

In its most basic characterization, a Flora can be defined as a taxonomic treatment of the plants of a clearly delimited geographic area (Meyer et al. 1997). It has also been defined as an inventory of plants occurring in a particular geographic region, providing a means to identify these plants (Diggs and Lipscomb 2002; Kirkup et al. 2005). This broad meaning includes other, less information-rich products that are not strictly Floras (e.g. a conspectus, which is similar to a Flora but lacks detailed descriptions, or even a catalogue with identification keys; see Meyer et al. 1997). A challenge faced by compilers of the WFO is deciding what fields to include in an online Flora as mandatory, and which should be optional.

In the traditional sense Floras typically include information on a broad range of organismic and environmental elements. The data content of a Flora, and consequently, an online Flora, can vary from having only a name, synonyms, descriptive text and possibly images, to those which extend to basionyms, homonyms, distribution and habitat, endemism, identification keys, etc. (Smith 2005; eFloras 2008).

At the national level, countries will decide what floristic elements should be included and what the requirements of the end-users will be (Morin et al. 1988; Smith 2005; Victor et al. 2013). The floristic information provided by the independent institutions or countries will therefore not be consistent and not all regions would be able to provide the same level of information. Most regions will rely heavily on previously published litera-ture. It is therefore necessary to decide at a global level which elements are rudimentary for each taxon to qualify as an entry into the WFO and which elements will enhance the quality of the product. At the 16th meeting of the Subsidiary Body on Scientific, Technical and Technological Advice (SBSTTA) of the CBD, certain core fields were suggested as being necessary to produce a WFO, with supplementary fields being regarded as optional (CBD 2012). These core fields included the accepted scientific name, family and synonyms, description, distribution, images, keys and references; supplementary fields included native status, habitat, altitude, conservation status, website links and vernacular names. This is, however, a work in progress, and the mandatory and optional fields are currently under discussion by the Technical Working Group of the WFO project. 
The third challenge is to ensure that there are links to, and interoperability with, other databases. The framework of WFO should be created in such a way as to be compatible with the data standards used to share information with other platforms such as EOL and GBIF. The information that WFO will provide will have similarities to that provided by EOL and GBIF although, currently, these systems are not substantial in terms of the descriptive information provided. GBIF is somewhat more focused on spatial (locality) data than EOL (which obtains its maps from GBIF). The two systems share distribution information and are cross-referenced on both sites. WFO will also be able to share information if the same data standards are used throughout all these systems. Links from WFO to specimen data held in GBIF will be particularly valuable to users seeking more precise distribution data.

\section{Methods for achieving a national online Flora}

In a broad sense, the compilation of a national online Flora for South Africa will inevitably follow a similar methodology as used to produce a WFO. The process will be initiated by using SANBI's checklist of South African flora published by Germishuizen et al. (2006), that is maintained in an electronic database and continually updated, as the taxonomic backbone. South Africa is in a privileged position to have regional floristic treatments that have either been published or that are currently being compiled by a dedicated team of taxonomists. These treatments can be used to extract valuable floristic information that can be consolidated to form the major part of the online Flora of South Africa (e-Flora).

Compiling the e-Flora will involve the incorporation of all names from the checklist into a relational database where the synonyms are linked to the accepted names. Available floristic information will then be harvested from regional Floras through markup and stored in the database. A time-frame for the compilation of the e-Flora is provided in Table 1.

Certain elements are commonly included in floristic treatments (Smith 2005; eFloras 2008; Rilke et al. 2012). Therefore we have selected the following elements that are in agreement with the majority of Floras as fields that should and can be accommodated in order to provide authoritative information to support research and conservation that facilitates the implementation of the CBD. Including these elements in an online Flora for South Africa is regarded as a realistic target to achieve by 2020 :

- up-to-date names with authorship for all taxa within South Africa (covering family, genus, species and infraspecific names);

- protologue citation;

- $\quad$ synonyms (including widely used taxonomically alternative names);

- descriptions;

- identification keys to the families, genera and where available to speciesinitially available text (dichotomous) keys should be referenced, but with an aim to move to multi-access keys and other options as technology, markup, and data parsing become available. Inclusion of keys may be facilitated by linkages to existing sources such as http://www.identifylife.org/ or online Flora projects;

- images - photographs and/or drawings of at least one species of each genus (ideally, linked to voucher specimens) should be included where available. 
Alternatively an imaged herbarium specimen or link to the URL of the type specimen (if available) should be provided.

- geographic scope - national-level geographic distribution ranges (with accompanying maps), or within floristic regions;

- resident status - taxa will be flagged as, e.g., exotic/alien, indigenous/native, naturalised;

- endemic status - taxa that are endemic will be flagged; and

- literature references and credits - attribution of authors, sources of data and images included in the Flora.

- Supplementary fields, which could be added subject to availability of resources and time, include:

- links to higher-level classification (such as order);

- categories of habit/life form

- categories of habitat and ecology, e.g. altitudinal range-although usually part of the description of a geographic range, general categories can be added to provide coarse sorting of plant species;

- specimen citations;

- links to digitised specimen information;

- conservation status;

- vernacular names - to be included if available;

- traditional, economic, and medicinal uses;

- trade statistics and non-detriment information for implementing the Convention on International Trade in Endangered Species of Wild Fauna and Flora (CITES);

- invasiveness - potential or actual;

- online links - to external data and data sources about the species, e.g. DNA barcodes; and

- a system that enables submission of feedback that can be included or acted upon to correct or improve the content.

The technological resources available today have changed the emphasis of certain components in a Flora and there are several key advantages in publishing electronic Floras. Previously, descriptions and dichotomous identification keys were the bases for identification. Nowadays, emphasis can be given to visual data, such as specimen images, field photographs and multi-access keys (see Koekemoer and Steyn 2009). Electronic Floras are dynamic systems that can easily and continuously be improved without incurring the same costs as for paper-published Floras. Additionally, electronic Floras can be updated rapidly and dynamically in comparison to the time it takes to publish in hardcopy format. However, there will inevitably be costs involved in maintaining and updating an online Flora. Names of taxa change regularly for nomenclatural or taxonomic reasons and such changes must be incorporated and referenced to ensure that the content of the Flora is kept current. This requires the availability of broadly-based taxonomic expertise - a rare commodity — along with biodiversity informatics specialists who can develop and maintain a robust electronic shell into which content is cast and from which it is served on the worldwide web. Committing to producing an online Flora is not a once-off endeavour: it will require resources. As technology advances, e-Flora information can potentially be searched through multiple access points on a tablet computer or mobile phone whereas it is 
impractical to carry around numerous volumes of heavy weight Floras (Brach and Boufford 2011). In addition, there are increasing technological advances in using DNA barcoding as an identification mechanism, for example BOLD Systems (http://www.boldsystems.org/).

The compilation of a traditional Flora is time consuming and not achievable within the set timeframe of the GSPC 2011-2020. It generally takes approximately two days for an experienced taxonomist to compile a comprehensive species treatment for a known taxon, including related distribution and habitat information (Gomez-Pompa and Nevling 1988). Because of the short timeframe, it is not feasible to undertake new taxonomic revisions and write new keys and descriptions for the c. 20,500 plant species found in South African or for the world's c. 400,000 plant species. Therefore, initially the Flora aims to be synoptic, populated by pre-existing data on as many of the plant species of the world as possible (CBD 2012). Once this foundation is in place, it will be possible to identify gaps in the data. Filling these gaps will be a challenge that can be met at a national level by development of biosystematics research strategies and building capacity.

In response to SANBI's mandate to "coordinate and promote the taxonomy of South Africa's biodiversity', a national biosystematics research Strategy has been developed (Victor et al. 2013). The Strategy highlights taxonomic research priorities for algae, animals, bacteria and archaea, fungi and plants. The first objective of the plant systematics component of the Strategy proposes that an online Flora of South African plants can be achieved using existing species descriptions, in particular those that are available in recently published family treatises or generic revisions.

Descriptions that are not published in family-level treatments or generic revisions can be extracted from regional Floras, published by SANBI, which will have covered the entire country before 2020. The South African National Plant Checklist will be updated by SANBI and lodged on the Institute's website, forming the backbone of the national e-Flora. The second objective of the Strategy identifies gaps in taxonomic information, in terms of revisions of plant genera that are outdated or inadequate, and prioritises which groups most urgently require attention (Victor and Smith 2011). These revisions can be used to populate the e-Flora on a continuous basis as they are produced. The third objective of the Strategy is to resolve taxonomic problems in plants of economic and conservation importance, where the genus to which the plant belongs is not necessarily in need of revision. The three strategic objectives should be met within a five-year time frame to provide a solid foundation for an online Flora.

The following procedure is a broad outline of SANBI's action plan to produce an eFlora for South Africa by 2020, to contribute to the WFO:

\section{Resources required}

SANBI has appointed one full-time trained taxonomist (e-Flora Co-ordinator) to coordinate the South African component of the WFO, with the help of at least one fulltime assistant. SANBI has to ensure that sufficient infrastructure and information technology resources are available to support this function. In addition to cost of employees, further financial input is required for travel both locally and internationally in order for the e-Flora Co-ordinator to liaise with external 
contributors to the project as well as the WFO Technical Working Group. External collaborators will be approached to contribute information at their own cost.

\section{Selection of an appropriate data management system}

It is important to decide on a system that will fulfil all the requirements to build an eFlora. The system should be able to accommodate and manipulate information (core fields as well as supplementary fields) at different taxonomic levels (family, genus, species, etc.). It would be an advantage if the system could manage and incorporate information taken from individual herbarium specimens, e.g. for the production of maps. Other functions that should be considered when selecting an appropriate system include the ability to (a) manipulate and update large sets of data and (b) to communicate with other information systems using biodiversity information standards such as Darwin Core Archive (Biodiversity Information Standards 2009) to export information into an appropriate format in order to contribute to projects such as the WFO.

The Botanical Research And Herbarium Management System (BRAHMS; Filer 2012) is capable of incorporating and manipulating inputs and delivering all the outputs necessary to build the e-Flora of South Africa. The software has the ability to combine information from many different fields, e.g. information gathered from herbarium specimens, botanical surveys, living collections, seed banks, literature from several sources with corresponding references, images, keys, etc. (Filer 2012). SANBI has recently adopted BRAHMS to house all specimen data, and will therefore also use BRAHMS to house all taxonomic data from where the floristic information will be parsed onto a website where the e-Flora of South Africa will be published.

The advantage of using BRAHMS is the interoperability of the database, which enables countries to share data. For example South Africa would be able to provide information to neighbouring countries with species in common. Another advantage of BRAHMS is that it is widely available and accessible online. For countries that are not able to use BRAHMS as their Floristic data management system, a simple database can be created using for example Microsoft Access or even Excel.

\section{Populating the database}

All core fields are populated for each taxon. Initially, descriptions from regional treatments will be incorporated; later on, they will be supplemented by descriptions from taxonomic revisions.

Family and genus descriptions are already available for most monocotyledons, dicotyledons and gymnosperms in South Africa, published by SANBI in Seed plants of southern Africa (Leistner 2000). In addition, SANBI is currently in the process of publishing regional floras for all provinces of South Africa: KwaZulu-Natal; Free State; Eastern Cape; Western Cape (Manning and Goldblatt 2013; Snijman 2013); Northern Cape; and the northern provinces comprising Gauteng, Limpopo, Mpumalanga and North West (Retief and Herman 1997, with plans to update by 
2018). These regional Flora treatments will serve as the basis for the e-Flora (see Table 1 for the estimated time-frame of the project). Furthermore, almost $20 \%$ of South Africa's flora has been treated at family level in a project initiated in 1955, The Flora of Southern Africa project. The aim was to document more than 20,000 species of 180 angiosperm families in South Africa, Lesotho, Swaziland, Botswana and Namibia. Due to slow progress, the project was de-emphasised in 1995 in favour of publishing family treatises in SANBI's in-house journal, Bothalia, a practice that is still currently active. Descriptions from these family treatises will be used in place of the regional Flora information where they are more recent or of a higher standard. Finally, recently published revisions of plant genera may be used if published in a SANBI journal, or if consent can be obtained from the publishing journal and authors (Sierra et al. 2013). Attribution is considered to be a very high priority for all data used, especially descriptions and images, considering that many taxonomists from around South Africa, and possibly internationally, are expected to contribute.

The technical process of populating the database includes the following steps:

a. Digitisation and markup

Literature published only in hard copy format has to be converted to digital format (Kirkup et al. 2005). Converting literature requires the scanning of each page, running it through optical character recognition (OCR) software (Pro-iBiosphere), converting the .pdf file into a Word document and editing the text for typos or unrecognised symbols and characters. Thereafter it has to be marked up for transfer into the database. Markup is mostly done manually where after the semantics has to be revised to ensure the import of data takes place correctly (Brach and Song 2006). It is time consuming and requires some basic botanical knowledge (Sierra et al. 2013).

b. Importing of data

Marked up literature is converted from .docx format, in Microsoft Word, to plain text after which it is imported into Microsoft Excel or Access. The best set of information (core elements) has to be evaluated from all literature resources of which the best quality data is imported into BRAHMS.

c. Inclusion of images

Images will be uploaded into BRAHMS through the Image Management System. Details of contributors or contributing institutions and any related copyright will clearly be linked to each image (whether these are photographs, illustrations or scans).

d. Keys

Keys to family and genus level will initially be taken from Leistner's publication (2000) and will be updated to the same classification system according to which the SANBI herbaria (NBG, NH and PRE) are arranged. 
e. Updates to the taxonomic backbone

The taxonomic backbone for the South African flora will be held in BRAHMS and will be updated on an on-going basis. As updates are made, floristic information will be incorporated for the new taxa.

\section{f. Quality control}

Before any data are published online, they will be verified. Once published online, provision will be made for users to provide feedback in order to improve the quality of the data.

\section{Disseminating and publishing the e-Flora}

According to the 16th meeting of SBSTTA (CBD 2012), the primary technical challenge for the WFO is to develop an open-access web-based facility for data input, manipulation, storage, and delivery, with the functionality to tailor outputs as desired by end-users, e.g., for national or regional contexts, particular plant families or genera, specific kinds of organisms (such as trees, conifers, mosses), or threatened species. The database should be easily able to permit editing by approved specialists, and must be compatible with a wide variety of external hardware and software options (CBD 2012).

BRAHMS has the capability of exporting information directly to a word processor, which enables easy editing and proofreading of the text (Filer 2012).The Biodiversity Information Management Directorate (BIM) within SANBI will assist with the develop-ment of the e-Flora website in South Africa. All information relevant to the e-Flora will be exported from BRAHMS onto the website using the BRAHMS publishing function (BRAHMS online) and will be made available to the WFO accompanied by metadata.

Once the initial aim of Target 1 of the GSPC 2020 has been accomplished and all core fields are published online, supplementary fields will be populated with the relevant information. Given the dynamic nature of taxonomic work, often leading to changes in names and their status, and the steady accumulation and refinement of our knowledge of species (Constance 1964), it is important that both the database and the website are maintained and updated.

\section{Future work}

After the first phase of the development of the e-Flora is completed (with the aim of submitting a national Flora to WFO by 2020), attention can be given to improving detail and providing consistent high resolution information, e.g.

(a) Create comprehensive species descriptions for all taxa using a template.

(b) Provide at least one image for each species and where possible photos of taxa in situ showing diagnostic characters. 
(c) Create interactive species-level keys.

\section{Conclusion}

In South Africa it will be possible to use existing resources to compile an online Flora. The availability of these current resources is a result of commitment and collaborative efforts over many years to enhance botanical knowledge in this country. All countries can benefit from data at regional or global level even where there has been no great national invest-ment (as there has been in South Africa). In this way, national implementation can be greatly enhanced by use of existing material and judicious targeting of resources to fill the gaps that are most critical to the conservation priorities of the country.

The WFO will be a critical resource for management and conservation of plants by providing baseline information on plant diversity for each region or country, enabling effective identification, clearly documenting geographic distributions, and supporting all other GSPC Targets, including those likely to be set in the decades beyond 2020 .

Achieving a World Flora describing some 400,000 species by 2020 may seem unrealistic, but there is an enormous body of usable data available, scientists with expertise, a formal recognition that such a Flora is a priority and urgently needed, and a broad-based enthusiasm to make it happen. Concerted efforts from many contributors in the world wide taxonomic community, along with leadership from key institutions, will be required to achieve this target, so that taxonomists are able to have a positive impact on global conservation efforts by providing the ultimate botanical resource. We urge other countries to consider how they might practically organise themselves to help deliver this challenging conservation target.

\section{References}

Biodiversity Information Standards (TDWG) (2009). ttp://rs.tdwg.org/dwc/index.htm

Brach AR, Boufford DE (2011) Why are we still producing paper Floras? Ann Missouri Bot Gard 98(3):297-300

Brach AR, Song H (2006) eFloras: New Directions for Online Floras Exemplified by the Flora of China Project. Taxon 55(1):188-192

CBD (2012) Subsidiary Body on Scientific, Technical and Technological Advice (SBSTTA) Global strategy for plant conservation: World flora online by 2020, 16th Meeting, Montreal, Canada. In: Convention on Biological Diversity, UNEP/CBD/SBSTTA/16/INF/38. http://www.cbd.int/doc/meetings/sbstta/sbstta16/information/sbstta-16-inf-38-en.doc

CBD (2013) Aichi Biodiversity Targets. http://www.cbd.int/sp/targets

Constance L (1964) Systematic botany_ an unending synthesis. Taxon 13:257-273 
COP (2002) Conference of the Parties (COP) Decision VI/9, Global Strategy for Plant Conservation, 2002-2010. In: Sixth ordinary meeting of the conference of the parties to the convention on biological diversity (COP 6), The Hague, Netherlands. http://www.cbd.int/decision/cop/?id=7183

COP (2010) Conference of the Parties (COP) 10 Decision X/17 (2011-2020). http://www.cbd.int/decision/ cop/default.shtml?id=12283

Diggs GM, Lipscomb BL (2002) What is the writer of a flora to do? Evolutionary taxonomy or phylogenetic systematics? Sida 20:647-674

eFloras (2008) Missouri Botanical Garden, St. Louis, Missouri, United States of America \& Harvard University Herbaria, Cambridge, United Kingdom.

http://www.efloras.org. Accessed 16 Oct 2013 Filer DL (2012) Botanical research and herbarium management system training guide. Department of Plant Sciences, University of Oxford, Oxford, United Kingdom

Germishuizen G, Meyer NL, Steenkamp Y, Keith M (eds) (2006) A checklist of South African plants. Southern African Botanical Diversity Network Report 41. SABONET, Pretoria

Gomez-Pompa A, Nevling, LI (1988) Some reflections on floristic databases. Symposium tropical botany: principles and practice. Taxon 37(3):764-775

Hamer M, Victor J, Smith GF (2012) Best practice guide for compiling, maintaining and disseminating national species checklists, version 1.0. Global Biodiversity Information Facility, Copenhagen. Accessed Oct 2012. http://www.gbif.org/orc/?doc_id=4752

IBC (2011) XVIII International Botanical Congress, July 2011 resolutions. http://www.ibc2011.com/downloads/resolutions\%20final\%2030\%20jul.pdf

Kirkup D, Malcolm P, Christian G, Paton A (2005) Towards a digital African Flora. Taxon 54:457-466 Klopper RR, Smith GF, Chikuni AC (2002) The global taxonomy initiative in Africa. Taxon 51:159-165 Koekemoer M, Steyn HM (2009)

Helichrysums of southern Africa. An interactive key to identification of the species. http://keys.lucidcentral.org/keys/v3/helichrysum/

Leistner OA (ed) (2000) Seed plants of southern Africa: families and genera.

Strelitzia 10. National Botanical Institute, Pretoria

Manning J, Goldblatt P (2013) Plants of the Greater Cape Floristic Region: The Core Cape Flora. Strelitzia 29: National Botanical Institute, Pretoria

Meyer NL, Mossmer M, Smith GF (eds) (1997) Taxonomic literature of southern African plants. Strelitzia 5. National Botanical Institute, Pretoria 
Morin NR, Whetstone RD, Wilken D, Tomlinson KL (1988) Floristics for the 21st century. In: Proceedings of the workshop sponsored by the American Society of Plant Taxonomists and the Flora of North America Project. Alexandria, Virginia

Paton A (2009) Biodiversity informatics and the plant conservation baseline. Trends Plant Sci 14:629-637 Paton AJ, Nic Lughadha E (2011) The irresistible target meets the unachievable objective: what have 8 years of GSPC implementation taught us about target setting and achievable objectives? Bot J Linn Soc 166:250-260

Retief E, Herman PPJ (1997) Plants of the northern provinces of South Africa: keys and diagnostic characters. Strelitzia 6. National Botanical Institute, Pretoria

Rilke S, Najmi U, Schnittler M (2012) Contributions to “'E-Taxonomy' — a virtual approach to the Flora of Mongolia (FloraGREIF). Feddes Repert 123(3):219-232

Sierra S, Agosti D, Groom O, Guntsch A, Hagedorn G, Hoverkamp P, Be'nichou L, Morris R, Mota de Oliveria S, Penev L (2013) D.3.1 — towards a set of best practices on editorial policies for the curation and publication of fundamental biodiversity data and information in an e-environment. Ppro-iBio-

sphere_WP3_Naturalis_D3.1_VFF_31052013.pdf. http://www.proibiosphere.eu/documents/

Smith AR (2005) Floristics in the 21st century: balancing user-needs and phylogenetic information. Fern Gaz 17(3):105-137

Smith GF, Buys M, Walters M, Herbert D, Hamer M (2008) Taxonomic research in South Africa: the state of the discipline. S Afr J Sci 104:254-256

Snijman DA (ed) (2013) Plants of the greater cape floristic region 2: the extra cape flora. Strelitzia 30. South African National Biodiversity Institute, Pretoria

Steenkamp Y, Smith GF (2006) Introduction to a checklist of South African plants. In: Germishuizen G, Meyer NL, Steenkamp Y, Keith M (eds) A checklist of South African plants. Southern African Botanical Diversity Network Report 41, SABONET, Pretoria pp iv-ix

The plant list (2010) version 1. http://www.theplantlist.org/. Accessed 2 May 2013

Victor JE, Hamer M, Smith GF (2013) A Biosystematics Research Strategy for the Algae, Animals, Bacteria and Archaea, Fungi and Plants of South Africa 2013-2018. SANBI Biodiversity Series 23. South - African National Biodiversity Institute, Pretoria

Victor JE, Smith GF (2011) The conservation imperative and setting plant taxonomic research priorities in South Africa. Biodivers Conserv 20:1501-1505 
Wearn JA, Chase MW, Mabberley DJ, Couch C (2013) Utilizing a phylogenetic plant classification for systematic arrangements in botanic gardens and herbaria. Bot J Linn Soc 172:127-141 
